\title{
Pauci-Immune Glomerulonephritis associated with Granulomatosis with Polyangiitis
}

National Cancer Institute

\section{Source}

National Cancer Institute. Pauci-Immune Glomerulonephritis associated with

Granulomatosis with Polyangiitis. NCI Thesaurus. Code C123111.

Glomerulonephritis in the context of granulomatosis with polyang iitis in which antineutrophil cytoplasm antibody (ANCA) is almost always present. 